\title{
Analysis of the diagnostic presentation profile, parathyroidectomy indication and bone mineral density follow-up of Brazilian patients with primary hyperparathyroidism
}

U.E.M. Oliveira ${ }^{1}$, M.N. Ohe ${ }^{1}$, R.O. Santos ${ }^{2}$, O. Cervantes ${ }^{2}$, M. Abrahão ${ }^{2}$, M. Lazaretti-Castro ${ }^{1}$, J.G.H. Vieira ${ }^{1,3}$ and O.M. Hauache ${ }^{1,3}$
${ }^{1}$ Disciplina de Endocrinologia e Metabologia,

${ }^{2}$ Disciplina de Otorrinolaringologia e Cirurgia de Cabeça e Pescoço, Escola Paulista de Medicina, Universidade Federal de São Paulo, São Paulo, SP, Brasil

${ }^{3}$ Instituto Fleury, São Paulo, SP, Brasil
Correspondence

O.M. Hauache

Disciplina de Endocrinologia e

Metabologia, EPM, UNIFESP

Rua Pedro de Toledo, 781, 12 andar

04039-032 São Paulo, SP

Brasil

E-mail: omar.hauache@fleury.com.br

Research supported by CNPq and Instituto Fleury.

Received May 16, 2006 Accepted January 3, 2007

\section{Abstract}

Primary hyperparathyroidism is an endocrine disorder with variable clinical expression, frequently presenting as asymptomatic hypercalcemia in Western countries but still predominantly as a symptomatic disease in developing countries. The objective of this retrospective study was to describe the diagnostic presentation profile, parathyroidectomy indication and post-surgical bone mineral density follow-up of patients with primary hyperparathyroidism seen at a university hospital. We found 115 patients (92 women, median age 56 years) with primary hyperparathyroidism diagnosed during the last 20 years. We defined symptomatic patients based on the presence of any classical symptom affecting bone, kidney or the neuromuscular system. Surgical criteria followed the guidelines of the National Institutes of Health regarding asymptomatic primary hyperparathyroidism. Symptomatic patients and patients meeting surgical criteria for parathyroidectomy were 66 and $93 \%$ of the sample, respectively. Median calcium and parathyroid hormone values were $11.9 \mathrm{mg} / \mathrm{dL}$ and 189 $\mathrm{pg} / \mathrm{mL}$, respectively. After surgical treatment, $97 \%$ of patients were cured, with increases in bone mineral density of $19.4 \%$ in the lumbar spine and $15.7 \%$ in the femoral neck 3 years after surgery. Greater bone mass increases were detected in pre-menopausal women, men, and in symptomatic and younger patients, both in the lumbar spine and femoral neck. Our results support the previous findings of a predominantly symptomatic disease with a presentation profile that could be mainly related to a delayed diagnosis. Nevertheless, genetic and racial backgrounds, and nutritional factors such as calcium and vitamin $\mathrm{D}$ deficiency may play a role in the clinical presentation of primary hyperparathyroidism of Brazilian patients.
Key words

- Primary hyperparathyroidism

- Hypercalcemia

- Clinical presentation

- Parathyroidectomy

- Bone mineral density 


\section{Introduction}

Primary hyperparathyroidism (PHP) is an endocrine disorder characterized by hypercalcemia and elevated or inappropriately normal levels of parathyroid hormone. In its early descriptions, the classical disease was associated with involvement of the kidneys (renal calculi and nephrocalcinosis), skeleton (osteitis fibrosa cystica, with pain, pathological fractures and deformity), and neuromuscular system (proximal neuromuscular weakness) (1). In the 1970's, after the introduction of automated and routine measurement of serum calcium levels in developed countries, several studies reported a sharp increase in the diagnosis of PHP (2). The increased incidence of PHP in these countries was associated with a significant change in the presentation of the clinical spectrum of the disease. Most cases were asymptomatic at diagnosis, as opposed to the classical disease description (3).

In this context, studies currently conducted in Western countries show that less than 20\% of the patients present symptomatic PHP, i.e., PHP with the early described classical features (4). Parathyroidectomy is indicated for these cases. Management guidelines deal specifically with the asymptomatic form of the disease. Following these guidelines, approximately $50 \%$ of the PHP patients will meet one or more criteria for parathyroidectomy, even though most of these patients are asymptomatic (5). In contrast to these findings, studies in developing countries like India, China, Brazil, and others still show a high incidence of symptomatic cases (6-9). Late diagnosis, related to limited access to routine measurement of calcium levels and/or poor recognition of PHP, as well as the possible high prevalence of vitamin $\mathrm{D}$ deficiency in these countries, may lead to a more symptomatic clinical profile (10). However, as we have demonstrated in a recent study, the proportion of symptomatic cases is gradually but still slowly decreasing in our public service (11).
We reviewed our PHP data regarding the clinical and laboratory findings before and after surgical treatment during the last 20 years. The main purposes of the present study were to analyze the diagnostic presentation and surgical indication profile of PHP and the recovery of bone mineral density (BMD) after parathyroidectomy in our patients, in São Paulo.

\section{Patients and Methods}

The study was retrospective and evaluated PHP patients followed at the Bone Metabolism Unit of the Federal University of São Paulo (UNIFESP). Reports of all patients with PHP diagnosed between 1985 and 2004 were reviewed. Since this is a reference service, most patients are referred to it by other specialties. The diagnosis of PHP was mainly based on calcium measurements ordered during the follow-up of menopausal patients or during the investigation of bone diseases and urolithiasis. Calcium measurement was not used as a routine screening method.

The diagnostic criterion for PHP was based on hypercalcemia (total calcium levels $\geq 10.5 \mathrm{mg} / \mathrm{dL}$ and/or ionized calcium levels above the upper normal reference range) associated with high or inappropriately normal intact parathyroid hormone levels (iPTH, normal range 10-65 pg/mL). Patients with renal insufficiency, defined as creatinine $>1.5$ $\mathrm{mg} / \mathrm{dL}$, were excluded when kidney disease was not related to PHP.

A total of 131 PHP patients were diagnosed during the last 20 years. Of this group, 16 were excluded, 5 due to the fact that PHP was related with multiple endocrine neoplasia type 1,3 because of familial isolated PHP, and 8 because of renal insufficiency that was not related to PHP.

The remaining 115 patients with sporadic PHP were classified as symptomatic or asymptomatic according to the presence of classical hyperparathyroidism findings, defined as clinical or radiological bone dis- 
ease, nephrolithiasis, proximal neuromuscular weakness, and hospital admission related to hypercalcemic complications. Asymptomatic patients were further classified into surgical and non-surgical according to the criteria established by the National Institutes of Health Workshop on Asymptomatic PHP $(12,13)$. Surgical criteria included age below 50 years, serum calcium concentrations $1 \mathrm{mg} / \mathrm{dL}$ above the upper normal limit, nephrolithiasis, 24-h urinary calcium excretion above $400 \mathrm{mg}$, and densitometric osteoporosis of the lumbar spine or hip. Patients satisfying at least one criterion were scheduled for parathyroidectomy, while the asymptomatic patients who did not meet surgical criteria were followed clinically. Patients classified as symptomatic fulfilled at least one such criterion and were all submitted to surgery. Data about perioperative and late postoperative complications as well as pathological findings were also reviewed.

Until 1993 ( $\mathrm{N}=21$ patients), parathyroid hormone levels were measured by an aminoterminal specific radioimmunoassay. As of 1994, iPTH levels were measured by an immunofluorimetric assay. The median parathyroid hormone values calculated in this study refer only to these iPTH data obtained by immunofluorimetric assay. Total calcium levels were measured by colorimetric assays and ionized calcium with an ion-specific electrode. Bone densitometry of the lumbar spine (L1-L4) and femoral neck was obtained by dual-energy X-ray absorptiometry (DEXA) using Hologic QDR 4500 (Waltham, MA, USA) and Lunar DPX (Madison, WI, USA) devices. Measurements were always performed with the same device for each patient. The coefficient of variation based on repeated measurements with a spine phantom was lower than $0.5 \%$ for both devices and the service coefficient of variation was $3.5 \%$ for both lumbar spine and femoral neck. Absolute Hologic BMD data were converted to Lunar data for calculation and comparison of mean and median parameters and for the determination of longitudinal changes. Conversion equations were as follows, as defined elsewhere (14):

$$
\begin{aligned}
& \text { Lunar } \mathrm{DPX}_{\text {lumbar spine }}=(1,074 \times \text { Hologic } \\
& \left.\mathrm{QDR}_{\text {lumbar spine }}\right)+0.054
\end{aligned}
$$

Lunar $\operatorname{DPX}_{\text {femoral neck }}=(1,013 \times$ Hologic $\left(\mathrm{QDR}_{\text {femoral neck }}\right)+0.142$

Patients were classified as osteoporotic when the BMD T-score was lower than -2.5 $\mathrm{SD}$ in relation to young adults from the DEXA manufacturer's database, according to the definition established by the World Health Organization.

\section{Statistical analysis}

Data are reported as median or means \pm SD. Statistical comparisons were performed using the Student $t$-test and the Mann-Whitney test for quantitative variables and the $\chi^{2}$ test for qualitative variables. BMD data were assessed by analysis of variance (ANOVA) to evaluate changes relative to baseline. A P

Table 1. Baseline clinical and laboratory data of patients with primary hyperparathyroidism.

\begin{tabular}{llc}
\hline & & \\
& & Reference range \\
\hline Women & $80 \%(92 / 115)$ & - \\
Age (years) (age range) & $56.0(11$ to 86$)$ & - \\
Symptomatic patients & $66 \%(74 / 112)$ & - \\
Patients with surgical criteria & $93 \%(107 / 115)$ & - \\
Osteoporosis & $71 \%(72 / 101)$ & - \\
Nephrolithiasis & $39 \%(43 / 111)$ & - \\
$24-h$ urinary calcium $\geq 400$ mg & $28 \%(27 / 96)$ & - \\
Calcium >1 mg/dL above the UNL & $59 \%(64 / 109)$ & - \\
Age $\leq 50$ years & $31 \%(36 / 115)$ & $8.5-10.5$ \\
Serum calcium (mg/dL) & $11.9(109 / 115)$ & $1.20-1.40$ \\
lonized calcium (mmol/L) & $1.52(101 / 115)$ & $10-65$ \\
iPTH (pg/mL) & $189.0(95 / 115)$ & $2.5-4.5$ \\
Serum phosphorus (mg/dL) & $2.1(102 / 115)$ & $80-250$ \\
Alkaline phosphatase (IU/L) & $254(100 / 115)$ & $0.6-1.5$ \\
Serum creatinine (mg/dL) & $0.9(106 / 115)$ & $60-220$ \\
$24-h$ urinary calcium (mg) & $308.0(91 / 115)$ & \\
& &
\end{tabular}

Data are reported as median or percentage. $\mathrm{UNL}=$ upper normal limit; $\mathrm{PPTH}=$ intact parathyroid hormone. 
value equal to or lower than 0.05 was taken to indicate a significant difference.

\section{Results}

The baseline clinical and laboratory data of PHP patients are reported in Table 1. Classical PHP signs and symptoms were detected in $66 \%$ of the sample. When compared to the asymptomatic patients, these symptomatic patients presented higher serum calcium, iPTH and urinary calcium levels and lower phosphorus levels (Table 2). However, even among the remaining asymptomatic patients, $79 \%$ also fulfilled at least one surgical criterion. Thus, as a whole, only $7 \%$ of these patients were not surgical candidates and $93 \%$ met at least one surgical criterion at presentation. The main surgical criteria were lumbar spine and/or femoral neck densitometric osteoporosis (71\%) and total calcium $1 \mathrm{mg} / \mathrm{dL}$ above the upper normal limit (59\%). Nephrolithiasis (39\%), age less than 50 years $(31 \%)$ and 24-h urinary calcium higher than $400 \mathrm{mg}$ (28\%) were less prevalent (Table 1).

\section{Clinical and densitometric follow-up of the operated patients}

Follow-up data were available for most of the 115 patients, $93 \%(\mathrm{~N}=107)$ were scheduled for parathyroidectomy, but only $84 \%(\mathrm{~N}=90)$ of them were operated. Patients were not operated either because their clinical conditions did not permit surgery or because they did not accept the procedure. These patients, as well as the asymptomatic patients who did not meet the surgical criteria, are currently being followed clinically.

Table 2. Comparison of laboratory and bone mineral density (BMD) parameters of symptomatic and asymptomatic patients with primary hyperparathyroidism.

\begin{tabular}{lccc}
\hline & Symptomatic patients & Asymptomatic patients & Reference range \\
\hline Serum calcium $(\mathrm{mg} / \mathrm{dL})$ & 12.7 & $11.2^{*}$ & $8.5-10.5$ \\
Parathyroid hormone $(\mathrm{pg} / \mathrm{mL})$ & 256.5 & $138.5^{*}$ & $10-65$ \\
Alkaline phosphatase $(\mathrm{IU} / \mathrm{L})$ & 264.5 & 245.0 & $80-250$ \\
Serum phosphorus $(\mathrm{mg} / \mathrm{dL})$ & 2.05 & $2.40^{*}$ & $2.5-4.5$ \\
24-h urinary calcium $(\mathrm{mg})$ & 332.0 & $242.0^{*}$ & $60-220$ \\
Lumbar spine BMD $\left(\mathrm{g} / \mathrm{cm}^{2}\right)$ & $0.823 \pm 0.200$ & $0.820 \pm 0.146$ & - \\
Femoral neck BMD $\left(\mathrm{g} / \mathrm{cm}^{2}\right)$ & $0.707 \pm 0.174$ & $0.739 \pm 0.131$ & - \\
\hline
\end{tabular}

Data are reported as median or means $\pm S D$.

${ }^{*} P<0.05$ compared to symptomatic patients (Mann-Whitney test).

Table 3. Bone mineral density (BMD) evolution of the operated patients with primary hyperparathyroidism at baseline and at 12 and 36 months after surgery.

\begin{tabular}{lccccccc}
\hline & $\begin{array}{c}\text { Baseline } \\
(\mathrm{N}=65)\end{array}$ & $\begin{array}{c}12 \text { months } \\
(\mathrm{N}=30)\end{array}$ & $\Delta 1$ & $\mathrm{P}^{*}$ & $\begin{array}{c}36 \text { months } \\
(\mathrm{N}=55)\end{array}$ & $\Delta 2$ & $\mathrm{P}^{+}$ \\
\hline $\begin{array}{l}\text { Lumbar spine BMD } \\
\left(\mathrm{g} / \mathrm{cm}^{2}\right)\end{array}$ & $0.823 \pm 0.188$ & $0.902 \pm 0.136$ & $9.6 \%$ & $<0.01$ & $0.983 \pm 0.169$ & $19.4 \%$ & $<0.01$ \\
$\begin{array}{l}\text { Femoral neck BMD } \\
\left(\mathrm{g} / \mathrm{cm}^{2}\right)\end{array}$ & $0.718 \pm 0.171$ & $0.795 \pm 0.130$ & $10.7 \%$ & $<0.01$ & $0.831 \pm 0.162$ & $15.7 \%$ & $<0.01$ \\
\hline
\end{tabular}

Data are reported as means \pm SD. $\Delta 1$ and $\Delta 2$ are the respective relative increases (in percent) in BMD at 12 and 36 months after surgery compared to baseline BMD.

${ }^{*}$ Compared to baseline BMD (ANOVA); ${ }^{+}$compared to baseline and to a 12-month period BMD (ANOVA). 
Of the operated patients, $30 \%$ needed intravenous calcium after surgery, $5 \%$ had transitory dysphonia and $2 \%$ had a cervical hematoma. After parathyroidectomy, all patients were discharged with a prescription of oral calcium and vitamin D supplements. The main pathological finding was parathyroid adenoma (77\%). Parathyroid hyperplasia and carcinoma were found in 10 and $9 \%$, respectively, while $4 \%$ had uncertain histopathological findings. Seventy-eight patients were followed up for a mean period of 47 months after surgery. Five patients $(6 \%)$ were successfully submitted to a second surgery because of a first non-curative surgery for a parathyroid adenoma. Almost all patients (97\%) submitted to parathyroidectomy were cured. Only $6 \%$ became permanently hypocalcemic. Five patients with histological parathyroid carcinoma with capsular and vascular invasion were normocalcemic dur- ing a mean follow-up period of 24 months after surgery, whereas three patients remained hypercalcemic and died during follow-up.

BMD increased significantly after surgery both in the lumbar spine $(9.6 \%$ in 12 months and $19.4 \%$ in 36 months) and femoral neck ( $10.7 \%$ within 12 months and $15.7 \%$ within 36 months), as shown in Table 3 . We analyzed these patients according to clinical parameters at presentation in order to find differences in bone mass evolution during the follow-up after surgery for a median period of 36 months (Table 4). All patient groups had significant increases in bone mass both in the lumbar spine and femoral neck. Asymptomatic patients who were not operated upon because they were not surgical candidates continued to have stable BMD during this period $(-1 \%$ in the lumbar spine and $4 \%$ in the femoral neck). When we

Table 4. Bone mineral density (BMD) evolution of primary hyperparathyroidism patients according to clinical parameters at baseline and after a median period of 36 months after curative parathyroidectomy.

\begin{tabular}{|c|c|c|c|c|c|c|c|c|c|c|}
\hline & \multicolumn{5}{|c|}{$\begin{array}{l}\text { Lumbar spine BMD } \\
\qquad\left(\mathrm{g} / \mathrm{cm}^{2}\right)\end{array}$} & \multicolumn{5}{|c|}{$\begin{array}{l}\text { Femoral neck BMD } \\
\left(\mathrm{g} / \mathrm{cm}^{2}\right)\end{array}$} \\
\hline & Baseline & 36 months & $\Delta$ & $P^{*}$ & $\mathrm{P}^{+}$ & Baseline & 36 months & $\Delta$ & $P^{*}$ & $\mathrm{P}^{+}$ \\
\hline $\begin{array}{l}\text { Premenopausal } \\
\text { women }(N=12)\end{array}$ & $0.875 \pm 0.277$ & $1.032 \pm 0.250$ & $18 \%$ & $<0.01-$ & & $0.707 \pm 0.289$ & $0.968 \pm 0.177$ & $37 \%$ & $<0.01]$ & \\
\hline $\begin{array}{l}\text { Postmenopausal } \\
\text { women }(N=42)\end{array}$ & $0.824 \pm 0.145$ & $0.929 \pm 0.118$ & $13 \%$ & $<0.01$ & 0.03 & $0.716 \pm 0.133$ & $0.784 \pm 0.144$ & $10 \%$ & $<0.01$ & $<0.01$ \\
\hline Men $(\mathrm{N}=11)$ & $0.758 \pm 0.218$ & $1.042 \pm 0.207$ & $38 \%$ & $<0.01$ & & $0.736 \pm 0.144$ & $0.878 \pm 0.128$ & $19 \%$ & $<0.01$ & \\
\hline $\begin{array}{c}\text { Age } \leq 50 \text { years } \\
(\mathrm{N}=22)\end{array}$ & $0.833 \pm 0.248$ & $1.076 \pm 0.188$ & $29 \%$ & $<0.01$ & 0.05 & $0.729 \pm 0.237$ & $0.931 \pm 0.167$ & $28 \%$ & $<0.01$ & 0.05 \\
\hline $\begin{array}{l}\text { Age }>50 \text { years } \\
(N=43)\end{array}$ & $0.817 \pm 0.152$ & $0.937 \pm 0.139$ & $15 \%$ & $<0.01$ & & $0.711 \pm 0.130$ & $0.782 \pm 0.137$ & $10 \%$ & $<0.01]$ & \\
\hline $\begin{array}{l}\text { Patients with } \\
\text { osteoporosis }(\mathrm{N}=49)\end{array}$ & $0.758 \pm 0.161$ & $0.954 \pm 0.172$ & $26 \%$ & $<0.01-$ & $<0.01$ & $0.661 \pm 0.151$ & $0.887 \pm 0.147$ & $34 \%$ & $<0.01]$ & 0.05 \\
\hline $\begin{array}{l}\text { Patients without } \\
\text { osteoporosis }(N=16)\end{array}$ & $1.020 \pm 0.114$ & $1.095 \pm 0.096$ & $7 \%$ & 0.05 & & $0.792 \pm 0.103$ & $0.975 \pm 0.134$ & $23 \%$ & $<0.01]$ & \\
\hline $\begin{array}{l}\text { Symptomatic patients } \\
\text { operated }(\mathrm{N}=35)\end{array}$ & $0.823 \pm 0.200$ & $1.017 \pm 0.174$ & $23 \%$ & $<0.01$ & & $0.707 \pm 0.174$ & $0.841 \pm 0.174$ & $19 \%$ & $<0.01$ & \\
\hline $\begin{array}{l}\text { Asymptomatic patients } \\
\text { operated }(\mathrm{N}=20)\end{array}$ & $0.820 \pm 0.146$ & $0.917 \pm 0.140$ & $12 \%$ & $<0.01$ & $<0.01$ & $0.739 \pm 0.131$ & $0.810 \pm 0.139$ & $10 \%$ & $<0.01$ & $<0.01$ \\
\hline $\begin{array}{l}\text { Asymptomatic patients } \\
\text { not operated }(\mathrm{N}=6)\end{array}$ & $1.065 \pm 0.161$ & $1.061 \pm 0.099$ & $-1 \%$ & 0.37 & & $0.861 \pm 0.189$ & $0.895 \pm 0.056$ & $4 \%$ & 0.85 & \\
\hline
\end{tabular}

Data are reported as means \pm SD. $\Delta=$ percent increase in BMD between baseline and 36 months after surgery.

${ }^{*}$ Compared to baseline BMD for each parameter (ANOVA); ${ }^{+}$comparison between groups regarding BMD increase 36 months after surgery (ANOVA). 
compared data between groups, we found greater bone mass increases in premenopausal women, in men, and in symptomatic and younger patients, both in the lumbar spine and femoral neck (Table 4).

\section{Discussion}

We observed that the pattern of clinical PHP presentation in our institution is mainly symptomatic, but we point out that only 115 patients were diagnosed between 1985 and 2004 at a reference public service. We think this low figure could be related to a delayed PHP diagnosis and to a still low recognition of the disease. In a city like São Paulo, with more than 10 million people, 115 patients diagnosed in 20 years at a reference service is far from the expected figure if we consider a prevalence of about 3/1000 in the general population (15). Most patients had a severe clinical and laboratory diagnostic presentation, probably as a result of several hypercalcemia complications owing to a late diagnosis. Perhaps only the more severe cases are referred to this service. The high prevalence of parathyroid carcinoma seen in our study could also be related to this selection bias, since it is much higher than the values reported in the Western literature (16), although similar prevalences have been found in other studies from developing countries $(6,17)$. Nevertheless, we emphasize that this presentation profile is gradually changing, as stated by Ohe et al. (11), who studied most of these patients and observed that the diagnostic incidence of PHP as well as the proportion of asymptomatic patients slowly increased in the last few years.

Due to the high prevalence of symptomatic cases $(66 \%)$, it was not a surprise that the majority of these patients were sent to surgery. Even among the remaining 38 asymptomatic patients, $79 \%$ still fulfilled surgical criteria. Consequently, only $7 \%$ of these patients were not scheduled for surgery ( 8 of 115 patients). These results agree with data obtained in other developing countries which also do not perform routine calcium measurements $(6,17)$. The biochemical parameters of symptomatic patients were more compromised at presentation than those of asymptomatic patients, but BMD was reduced in both groups, with no significant difference between them.

Considering the surgical guidelines proposed for asymptomatic patients with PHP, Silverberg and Bilezikian (5) reported that about $50 \%$ of these patients would meet at least one surgical criterion. Although we consider our high prevalence of symptomatic patients (66\%) and of surgical patients (93\%) to be mainly a consequence of delayed diagnosis, it is also possible that differences in the genetic and racial backgrounds of our population may play a role. These differences could be somehow modulated by local environmental factors such as low calcium intake and vitamin D deficiency. As a consequence of the high racial admixture of our population, it is extremely difficult, if not impossible, to appropriately separate the different Brazilian ethnic groups. Calcium and vitamin $\mathrm{D}$ deficiencies have been related to the clinical profile of PHP (18). Moosgaard et al. (19) demonstrated that vitamin D deficiency or insufficiency is a common finding in PHP patients and occurs more often than in gender- and age-matched controls. As observed in endemic areas of vitamin D deficiency, low levels of vitamin $\mathrm{D}$ could be related to a more aggressive clinical presentation of PHP, with greater adenoma size and overt bone disease $(10,15)$. Since we do not have data regarding the vitamin D status of our patients we cannot rule out this possibility.

As stated earlier, we observed a high prevalence of parathyroid carcinoma in our series, similar to those found in other series from developing countries $(6,17)$. Our cases were based on surgical findings such as large and poorly circumscribed tumors adherent to the surrounding soft tissues of the neck or 
of the thyroid gland and on pathological findings such as capsular and vascular invasion (20). Unfortunately, the follow-up of these cases is limited: three have died because of complications related to hypercalcemia and the remaining ones were followed for a mean period of only 24 months (21).

BMD determinations by DEXA provide a noninvasive and objective parameter of disease activity in PHP. In addition, available data indicate that DEXA can be used to classify patients with PHP and can be helpful regarding the decision about recommending surgery or not for individual patients. In our study we found a high prevalence of osteoporosis (71\%). Several studies have shown that regional BMD is decreased by 10 to $20 \%$ in untreated PHP patients, with greater involvement of the appendicular skeleton, which is compatible with a preferential cortical bone involvement in PHP (22). These densitometric studies as well as histomorphometric data have shown that high iPTH levels have a catabolic action on cortical bone and an anabolic action on trabecular bone, with relative preservation of bone mass in the lumbar spine (22). However, in our study, most of the osteoporotic patients had involvement of both femoral neck and lumbar spine BMD, with no preservation of lumbar spine BMD. Indeed, at presentation there were no differences in lumbar spine or femoral neck BMD between symptomatic and asymptomatic patients. In a 10-year follow-up study, $21 \%$ of asymptomatic PHP patients had a bone loss of more than $10 \%$ at one or more sites, whereas BMD was preserved in the remaining ones (23).

The remarkable BMD recovery observed in our patients after surgical treatment agrees with other studies. Silverberg et al. (24) found an increase of 12 to $13 \%$ in both lumbar spine and femoral neck BMD 4 years after parathyroidectomy. Nevertheless, it should be emphasized that these results were obtained for a majority of asymptomatic patients (76\%), with similar BMD compared to patients without surgical criteria in that study. Our patients differed in this context because, although symptomatic and asymptomatic patients meeting surgical criteria had similar BMD reductions, their reductions were greater than in patients without surgical criteria. Three years after surgery, our patients had BMD increases of $19.4 \%$ in the lumbar spine and of $15.7 \%$ in the femoral neck, and symptomatic patients had greater increases of BMD than asymptomatic patients (23 vs 12\% in the lumbar spine and 19 vs $10 \%$ in the femoral neck, respectively). Our asymptomatic patients also had significant increases in BMD, with results similar to those reported in the literature $(23,24)$. Given this and the fact that parathyroidectomy is a low-risk procedure, we conclude that asymptomatic patients meeting surgical criteria for parathyroidectomy (5) should be submitted to surgery.

In this retrospective study we reviewed data of PHP patients seen at our institution during the last 20 years, and our results support previous findings of a predominantly symptomatic disease. This PHP presentation profile could be mainly related to a delayed diagnosis and may reflect the fact that we are seeing only the more severe cases. Nevertheless, one should consider that genetic and racial backgrounds as well as nutritional factors such as calcium and vitamin D deficiency may play a role in the clinical presentation of PHP in Brazilian patients. The surgical treatment was successful in almost all the patients and recovery of BMD reached $19.4 \%$ in the lumbar spine and $15.7 \%$ in the femoral neck 3 years after surgery. 


\section{References}

1. Mallette LE, Bilezikian JP, Heath DA, Aurbach GD. Primary hyperparathyroidism: clinical and biochemical features. Medicine 1974; 53: $127-146$.

2. Mundy GR, Cove DH, Fisken R. Primary hyperparathyroidism: changes in the pattern of clinical presentation. Lancet 1980; 1: 1317-1320.

3. Wermers RA, Khosla S, Atkinson EJ, Hodgson SF, O'Fallon WM, Melton LJ III. The rise and fall of primary hyperparathyroidism: a population-based study in Rochester, Minnesota, 1965-1992. Ann Intern Med 1997; 126: 433-440.

4. Bilezikian JP, Potts JT Jr. Asymptomatic primary hyperparathyroidism: new issues and new questions - bridging the past with the future. J Bone Miner Res 2002; 17 (Suppl 2): N57-N67.

5. Silverberg SJ, Bilezikian JP. Evaluation and management of primary hyperparathyroidism. J Clin Endocrinol Metab 1996; 81: 2036-2040.

6. Biyabani SR, Talati J. Bone and renal stone disease in patients operated for primary hyperparathyroidism in Pakistan: is the pattern of disease different from the west? J Pak Med Assoc 1999; 49: 194198.

7. Bilezikian JP, Meng X, Shi Y, Silverberg SJ. Primary hyperparathyroidism in women: a tale of two cities - New York and Beijing. Int $J$ Fertil Womens Med 2000; 45: 158-165.

8. Marcinkowski W, Nieszporek T, Kokot F, Podwinski A, Niemiec A, Wieczorek M. Clinical and biochemical picture of primary hyperparathyroidism based on 155 observed cases. Pol Arch Med Wewn 2000; 103: 61-66.

9. Mishra SK, Agarwal G, Kar DK, Gupta SK, Mithal A, Rastad J. Unique clinical characteristics of primary hyperparathyroidism in India. Br J Surg 2001; 88: 708-714.

10. Rao DS, Agarwal G, Talpos GB, Phillips ER, Bandeira F, Mishra SK, et al. Role of vitamin $\mathrm{D}$ and calcium nutrition in disease expression and parathyroid tumor growth in primary hyperparathyroidism: a global perspective. J Bone Miner Res 2002; 17 (Suppl 2): N75-N80.

11. Ohe MN, Santos RO, Barros ER, Lage A, Kunii IS, Abrahao M, et al. Changes in clinical and laboratory findings at the time of diagnosis of primary hyperparathyroidism in a University Hospital in São Paulo from 1985 to 2002. Braz J Med Biol Res 2005; 38: 1383-1387.

12. Consensus development conference statement. J Bone Miner Res 1991; 6 (Suppl 2): S9-S13.
13. Bilezikian JP, Potts JT Jr, Fuleihan G, Kleerekoper M, Neer R, Peacock M, et al. Summary statement from a workshop on asymptomatic primary hyperparathyroidism: a perspective for the 21st century. J Bone Miner Res 2002; 17 (Suppl 2): N2-N11.

14. Genant HK, Grampp S, Gluer CC, Faulkner KG, Jergas M, Engelke $\mathrm{K}$, et al. Universal standardization for dual X-ray absorptiometry: patient and phantom cross-calibration results. J Bone Miner Res 1994; 9: 1503-1514.

15. Adami S, Marcocci C, Gatti D. Epidemiology of primary hyperparathyroidism in Europe. J Bone Miner Res 2002; 17 (Suppl 2): N18N23.

16. Marx SJ. Hyperparathyroid and hypoparathyroid disorders. N Engl J Med 2000; 343: 1863-1875.

17. Agarwal G, Mishra SK, Kar DK, Singh AK, Arya V, Gupta SK, et al. Recovery pattern of patients with osteitis fibrosa cystica in primary hyperparathyroidism after successful parathyroidectomy. Surgery 2002; 132: 1075-1083.

18. Miedlich S, Krohn K, Paschke R. Update on genetic and clinical aspects of primary hyperparathyroidism. Clin Endocrinol 2003; 59: 539-554.

19. Moosgaard B, Vestergaard P, Heickendorff L, Melsen F, Christiansen $\mathrm{P}$, Mosekilde L. Vitamin D status, seasonal variations, parathyroid adenoma weight and bone mineral density in primary hyperparathyroidism. Clin Endocrinol 2005; 63: 506-513.

20. DeLellis RA. Parathyroid carcinoma: an overview. Adv Anat Pathol 2005; 12: 53-61.

21. Vieira JG, Ohe MN, Hauache OM, Oliveira UM, Delana JM, Gonçalves $A$, et al. Parathyroid carcinoma. Arq Bras Endocrinol Metabol 2005; 49: 811-815.

22. Christiansen $P$. The skeleton in primary hyperparathyroidism: a review focusing on bone remodeling, structure, mass, and fracture. APMIS Suppl 2001; 1-52.

23. Silverberg SJ, Shane E, Jacobs TP, Siris E, Bilezikian JP. A 10-year prospective study of primary hyperparathyroidism with or without parathyroid surgery. N Engl J Med 1999; 341: 1249-1255.

24. Silverberg SJ, Gartenberg F, Jacobs TP, Shane E, Siris E, Staron $\mathrm{RB}$, et al. Increased bone mineral density after parathyroidectomy in primary hyperparathyroidism. J Clin Endocrinol Metab 1995; 80: 729-734. 